\title{
THE ROLE OF INTERACTIVE GRAPHICS WHEN APPLYING TRAFFIC SIMULATION MODELS
}

\author{
Edward B. Lieberman \\ Barbara Andrews \\ KLD Associates, Inc. \\ 300 Broadway \\ Huntington Sta., New York 11746
}

\begin{abstract}
This paper discusses the advantages of an interactive computer graphics (ICG) system that provides users with a new and highly efficient methodology for analyzing results generated by simulation models. An ICG tool that can be executed on microcomputers (i.e., PC's) or on graphics workstations greatly enhances the value of the simulation model by providing insights that cannot easily be obtained by other means.

To demonstrate the utility of ICG systems for simulation models, this paper outlines the concepts and objectives guiding the design of a tool named GTRAF which presents displays of results generated by a microscopic traffic simulation model named NETSIM. The reader is "walked-through" an ICG session with GTRAF, with several photographs of the displays shown to illustrate its value.
\end{abstract}

\section{INTRODUCTION}

For the most part, simulation models generally produce results in a statistical format. These results are then reviewed by the user to gain an understanding of the underlying process and to quantify the performance of the modeled system. When the system model is complex, it is likely that the statistical output generated by the model will be voluminous. Even though these data can be exported to packages such as spreadsheets for the purpose of creating graphical representations, the amount of effort to fully understand the operational characteristics of the system can be onerous.

To alleviate this problem, some high-level simulation languages incorporate graphical displays for presentation purposes which greatly assist the user in gaining an understanding of the underlying process. Primarily, these graphical displays present the data in a format which is useful.

Quite frequently the displays produced by such systems are inflexible in that the user cannot interact with them to abstract the information which is of greatest value. To counter this limitation, GTRAF was designed as an interactive graphics display program for a rather complex, general purpose, microscopic traffic simulation model named NETSIM. This software system, developed for the Federal Highway Administration and used widely throughout the country, is available on IBM compatible PC's.

The interactive computer graphics (ICG) tool is designed to read data generated for this purpose by the NETSIM model and to present displays in an interactive mode as requested by the user. While the discussion in this paper is limited to a description of the NETSIM model (1) and the ICG software, GTRAF, the technology is applicable for other simulation models.

NETSIM, which is probably the most popular and widelyused traffic simulation model in the U.S., is a Fortran-coded software program which describes the operational performance of vehicles traveling over a network of surface (i.e., non-access controlled) streets. The internal logic of this microscopic model describes the movements of individual vehicles responding to external stimuli including traffic control devices, the performance of other vehicles, pedestrian activity, transit operations and driver behavioral characteristics. Statistics are gathered which provide detailed traffic performance measures (e.g., speed, volume, density, delay, stops, intersection spillback, queuing, turn movements, fuel consumption, emission pollutants) on each network link, over specified time intervals. For more detailed description of NETSIM, see [Lieberman et al. 1985, Vols. 2-4].

As such, NETSIM is perceived as an engineering tool, both for research and for practical applications.

\section{NEEDS OF TRAFFIC ENGINEERING PROFESSIONALS}

Historically, the sharply decreasing costs of computation over recent years has been matched with an increasing cost of professional services. These divergent trends are taking place at the same time that there is a growing need for extracting improved productivity from a highway infrastructure of severely constrained growth, which must service a continually growing traffic demand.

Specifically, studies indicate that traffic demand is growing at a rate of $3 \%$ per year while construction of new facilities to accommodate this increased demand is increasing at a rate of much less than $1 \%$ per year. With $40 \%$ of urban travel already operating under congested conditions, at an annual cost of 37 billion hours, it is imperative that new and more productive tools be aggressively utilized to assist the engineer to improve traffic performance. In this environment, the engineer must achieve superior results in less time, with greater accuracy and reliability, and at lower cost, than ever before.

Fortunately, advances in computer technology and in available software systems offer the potential for developing the tools needed by engineers to cope with this situation.

\section{RESOURCES PROVIDED BY TECHNOLOGY}

These developments have, in turn, prompted FHWA to sponsor the development of software to greatly improve the user accessibility to these computer tools which have been "ported" from mainframes to microcomputers.

The use of graphics has spread rapidly in recent years as a preferred method of displaying and assimilating statistical data. Probably the most dramatic examples of the popularity of graphics are the emergence of computer-aided-design (CAD) and engineering (CAE) systems, the graphics presentations associated with such spreadsheets as Lotus 123 , and the explosive growth of graphics workstations designed expressly to service the engineering community. This transformation which presents results in a 
pictorial format instead of statistical tabulations provides impressive gains in engineer productivity.

With an ICG system the user can select the displays he/she wishes to view on a screen at any time during a "session" while seated at a microcomputer or terminal. Furthermore, the user need have no computer expertise whatever in order to access these tools; nor is there a need to learn a command vocabulary or to undergo formal training. The displays themselves are in color and are designed to provide all the information - and more which could otherwise be extracted, at great effort, from detailed listings of statistical tabulations.

\section{GTRAF - THE NETSIM GRAPHICS SYSTEM}

The effort to manually convert the statistical data generated by the NETSIM into information is time consuming and requires considerable skill. Consequently, this data assimilation and analysis effort is often the most costly aspect of an investigation employing the NETSIM model and most other simulation models. Because of this cost, and the need for skills which are in short supply, many agencies have not used the NETSIM model and have employed procedures of lesser utility.

Another concern of potential users of NETSIM is the issue of credibility. Many managers are reluctant to depend on a "black box" particularly if they feel uncomfortable - due to a lack of knowledge or understanding - with the simulation approach. It is often difficult for many to accept the idea that a computer tool can actually describe the complex processes of traffic flow at an acceptable level of accuracy and reliability. The GTRAF graphics software is designed to vividly demonstrate NETSIM's ability to realistically represent the traffic environment, thus addressing the credibility issue cited above.

The design objectives applied in the development of GTRAF are:

1. Eliminate the need for an extensive Users Guide or for substantive training by

a) Developing simple, "user-friendly" procedures for communicating with the graphics application software

b) Providing all guidance on-line in the form of brief "prompt" messages

c) Employing menus from which the viewer can select the next display, using either a mouse or allow keys.

d) Providing guidance to the user in the form of an on-line HELP feature, which explains how to apply GTRAF to analyze the problem at hand.

2. Utilize color as a means of transmitting graphical information.

3. Interleave text (numbers and words) with pictorial graphics to provide both qualitative and quantitative information.

4. Guide the viewer in identifying the problem areas (called "hot spots") in the traffic environment, quantifying their extent and analyzing the underlying cause-and-effect relationships.

5. Present animations of vehicle movements which impart to the user, a colorful "movie" depicting the dynamic traffic flow processes.

6. Perform all data management functions, which transform NETSIM output into GTRAF inputs, in a manner which requires no action on the part of the user.

7. Integrate NETSIM and GTRAF software to form a coherent system which enables users to perform all necessary functions in a single session.
8. Employ a graphics package which conforms with the GKS ANSI standard and develop all software exclusively in Fortran. Thus, the software can be implemented on any computer which supports an ANSI Fortran compiler and a GKS package, and which has a color monitor.

9. Provide hard copy of many graphical displays using a color plotter or, in black-and-white, a laser printer. These displays can be used for documenting a case study.

GTRAF was originally developed on an IBM PC AT. Currently, this software is operational on all PC's and performs exceptionally well on PC's with the INTEL $80386 \mathrm{cpu}$ and with either the Enhanced Graphics Adapter or Video Graphics Adapter (EGA and VGA), with a color monitor. The GKS graphics package used is a product of Graphics Software Systems, Inc.

As shown in the system configuration diagram in Figure 1, the NETSIM simulation program and the GTRAF graphics software are two independent pieces of software. NETSIM must be executed first to generate the data needed by GTRAF for the graphics displays. When executing NETSIM, a "case" name-identifier is specified as part of the input stream. The graphics data files produced during the NETSIM execution is then stored in files associated with the given case and the case name is entered in the case "log".

Thus, a "library" of case studies may be established and stored on disk. The results of any case entered in the case log may subsequently be viewed graphically using GTRAF.

The logical structure of the GTRAF display hierarchy is shown in Figure 2. GTRAF is subdivided into two parts: SNETG and ANETG. SNETG presents "static" graphical displays while ANETG presents the animation displays.

Upon entering the system, the user is presented with a "welcome" display which contains instructions on how to use the mouse (or cursor positioning keys) to select menu items and how to browse through the display hierarchy. The user then is provided with a "case-log" menu listing the various NETSIM output files which have been previously stored on hard disk. The user is prompted to select one of them. At this point, the software retrieves the selected file from disk and prepares the data for the ICG session.

The screen displays are usually configured as shown in Exhibit 1. The Menu Area contains the alternative actions that can be taken by the user to continue the interactive session. The Message Area contains a brief "prompt" statement that guides the user's next action, or offers timely information. The Main Viewport contains the display. On some screens, the menu area may be reduced, thereby permitting the main viewport to be expanded in size. The main viewport, itself, may be partitioned into several related displays.

\section{WALK-THROUGH OF AN ICG SESSION WITH GTRAF}

This section presents a "walk-through" of a portion of an ICG session, in order to illustrate the underlying procedures and features.

Exhibit 1, which displays the network schematic and the master menu, is the first display which appears after the viewer selects the NETSIM Case which is to be studied using GTRAF. The cross-hair cursor (not shown) can be moved using the mouse or cursor keys to any menu item. If the VIEW INPUT DATA menu item is PICKed (i.e., selected by clicking the mouse after the cursor is positioned over the selected menu item), then a new menu appears which offers a choice of displays. The viewer 
The Role of Interactive Graphics When Applying Traffic Simulation Models

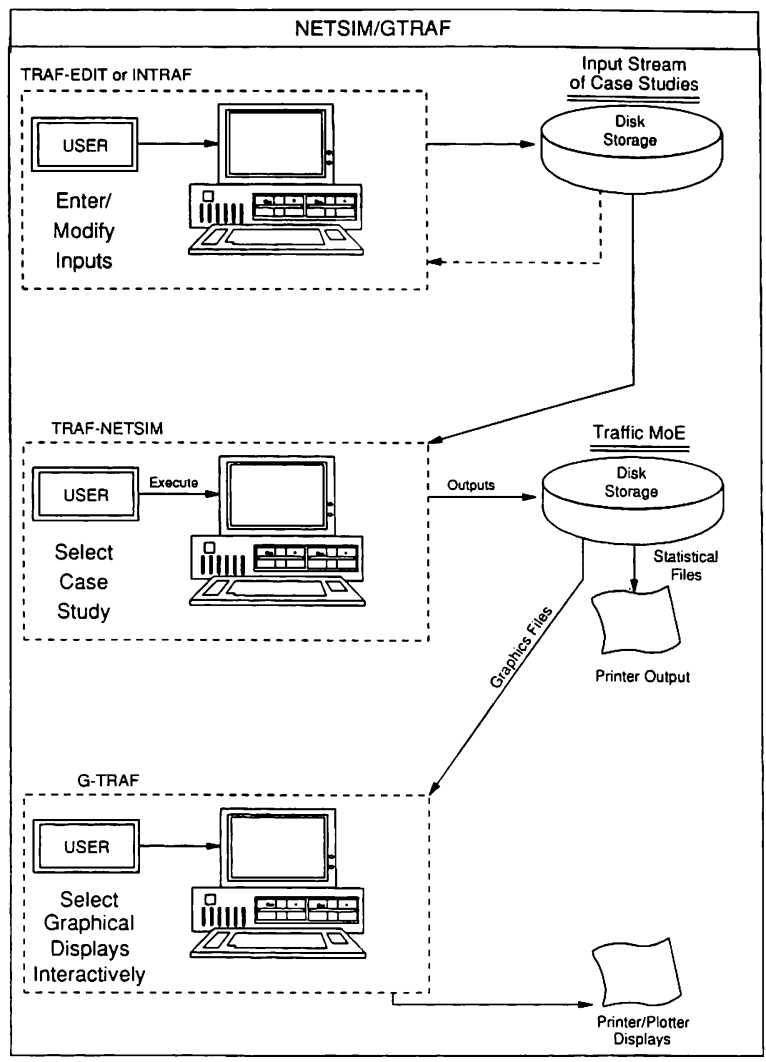

Figure 1. System Configuration

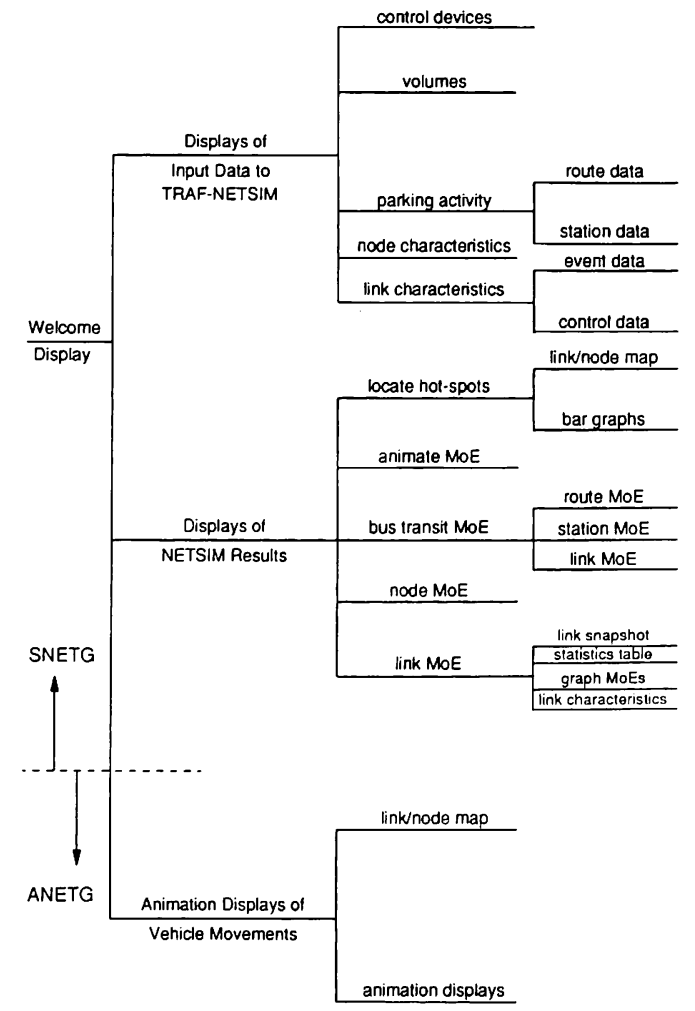

Figure 2. G-Traf Display Hierarchy

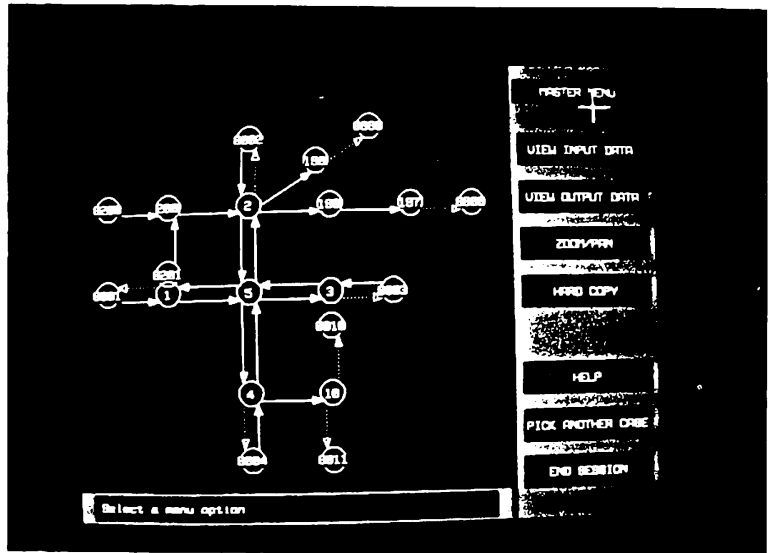

Exhibit 1. G-Traf Master Menu

could also elect, instead, to view the NETSIM output data, select another NETSIM case study or to end the session.

Note the "Prompt" message at the bottom of the screen which guides the viewer responses. These messages, the menus and the viewer responses (in the form of menu selections) constitute the mechanics of the man-machine interaction. The GTRAF software retains a display until action (i.e., a response to a prompt message) is taken by the user. This succession of user menuselection actions and GTRAF responses projecting the requested display on the screen, enables the user to "browse" at will through the NETSIM data base, extracting the information desired.

Whenever a display includes the network schematic, as in Exhibit 1, the ZOOM/PAN menu option is provided. The option enables the user to study sections of the network at a magnified scale or to view more of a network at a smaller scale, at any time. If this menu option is selected, a new menu appears which enables the user to pan left, right, up or down and/or zoom in or out, repeatedly, in any sequence.

Exhibit 2 is one of the displays available to a viewer wishing to review the NETSIM input stream. Here, the display presents the specified traffic demands at all Entry Nodes at the periphery and all internal Source/Sink nodes representing parking facilities. The different types of nodes are distinguished by different colors.

Exhibit 3 presents a detailed display of the links approaching and departing a selected node. This display indicates the lanes on all these links, a link-node sketch as a reference and the move-

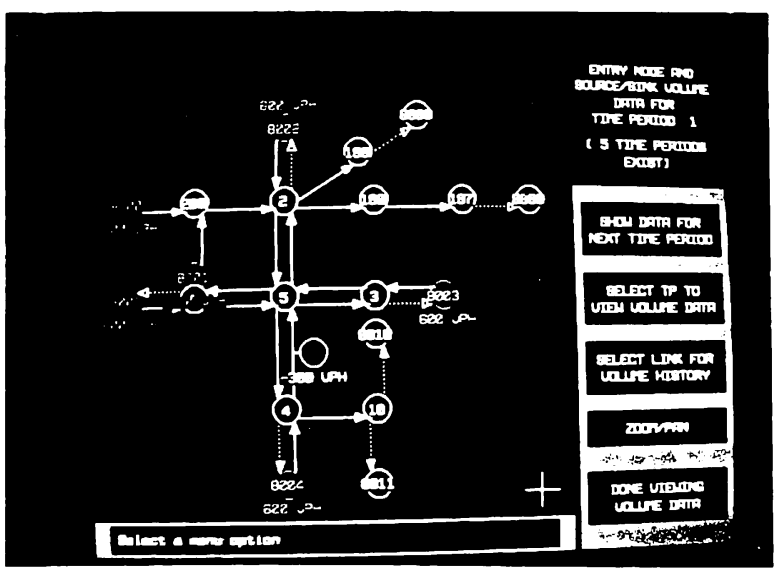

Exhibit 2. Volumes Display 


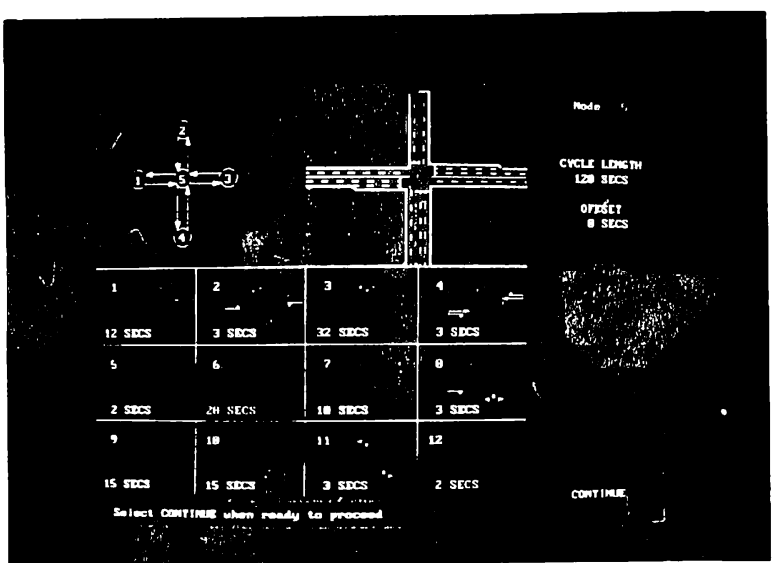

Exhibit 3. Interval Data Display

ments serviced during each signal interval. Each arrow depicting a traffic movement on an approach to the intersection is colored in red, green or yellow, defining the applicable control for the indicated signal interval. The signal cycle length, reference offset and each interval duration are also indicated. This Exhibit demonstrates how the Main Viewport can be subdivided into several related displays that integrate both graphics and data.

Exhibit 4 is a detailed link configuration showing the lane channelization, right-turn-on-red treatment, pedestrian volume, applicable signal control, specified turn movements and receiving nodes for each Time Period. Color is used extensively to distinguish the various features and signal control.

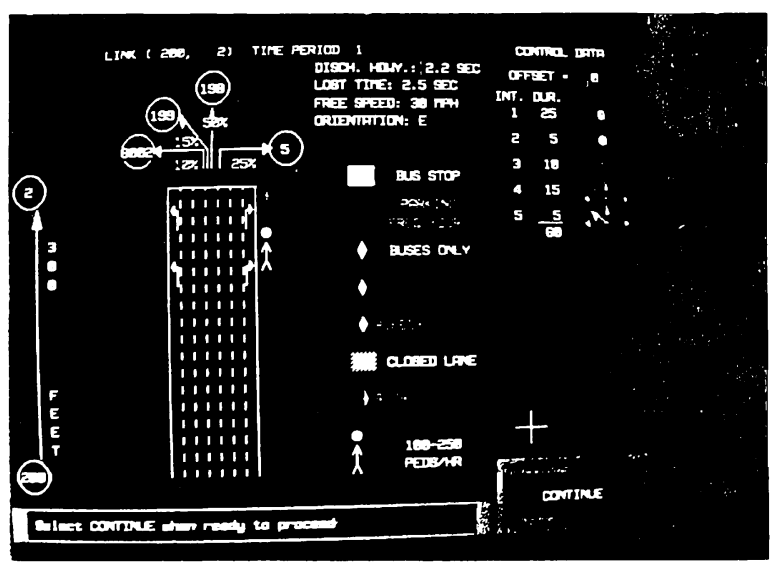

Exhibit 4. Link Characteristics Display

One of the most valuable features of GTRAF is the "Hotspots" menu option. Under this option, the viewer can request GTRAF to present displays which identify those links which exhibit extreme conditions, relative to the network as a whole. For example, the viewer can ask the system to identify those links with the lowest speed, greatest delay, highest volume or highest density, etc. With this feature, the viewer can immediately identify and analyze the "trouble-spots" within the network.

Exhibit 5 identifies the five links with the highest discharge volume in a link-node format. These links are highlighted in red and the values of vehicle trips for these links are presented numerically.

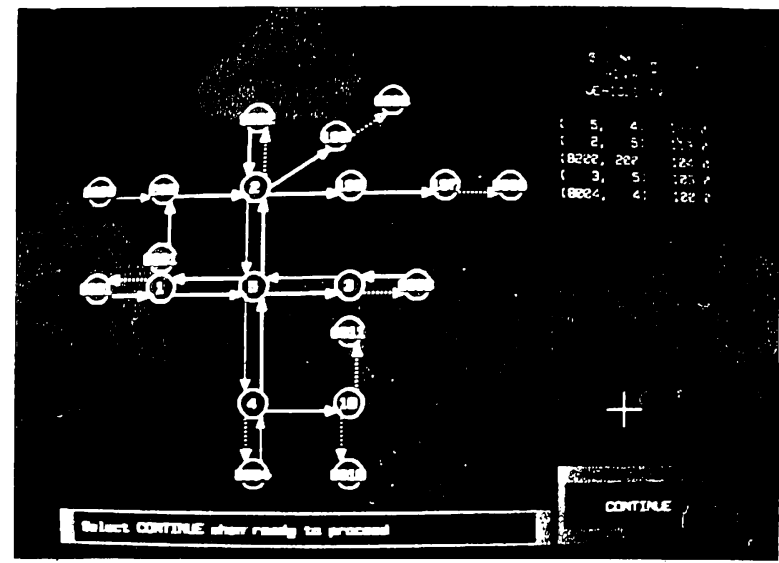

Exhibit 5. Hot-Spots Display (link/node format)

Exhibit 6 displays one format for presenting vehicle movements. Here, the vehicles on the approach links to the intersection are controlled by an actuated signal which responds to the volume of traffic. The legend is colored to indicate the various traffic signal phases. The user can step ahead (or back) in time to observe how the traffic environment changes with time. Note the time at which this "snapshot" is taken.

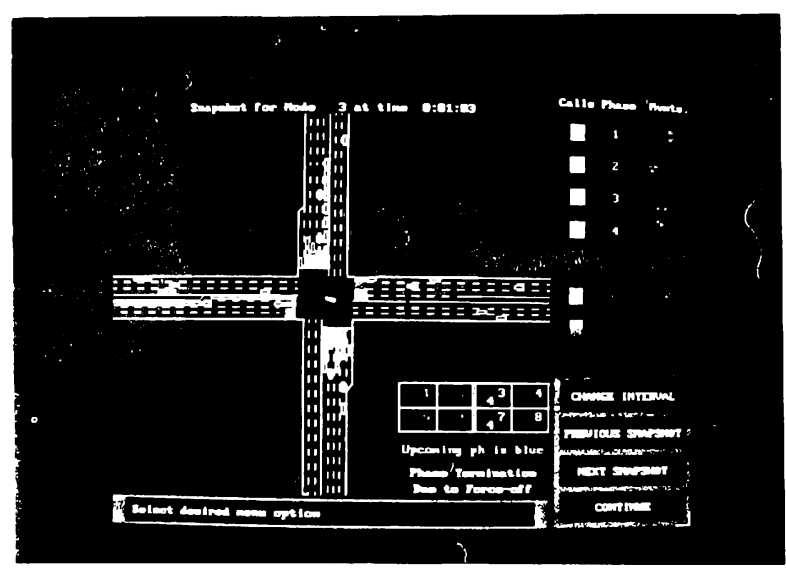

Exhibit 6. Node Snapshop Display

Exhibit 7 is a display of a wide range of statistics (Measures of Effectiveness, MoE) output by NETSIM for user-selected links, at selected time intervals. As indicated, GTRAF can provide extensive numerical data in adidtion to pictorial displays.

In addition to these static displays, GTRAF offers the additional option of vehicle animation at one-second (or less) time-steps over a selected portion of the network, for specified time intervals. That is, the user can get the same visual inputs from the screen as though he were observing the traffic flow from a stationary helicopter hovering overhead. The animation is performed on a schematic display of the network which is drawn to scale and shows curb lines, stop-lines, lane lines, median lines, turn pockets and signal indications. Thus, the vehicle movements and changing signal indications provide a graphical representation of the dynamic traffic environment. The user can control the animation to suit his objectives.

Exhibit 8 is a single frame from an animation display. All vehicles are shown in this "aerial" view as red rectangles that 


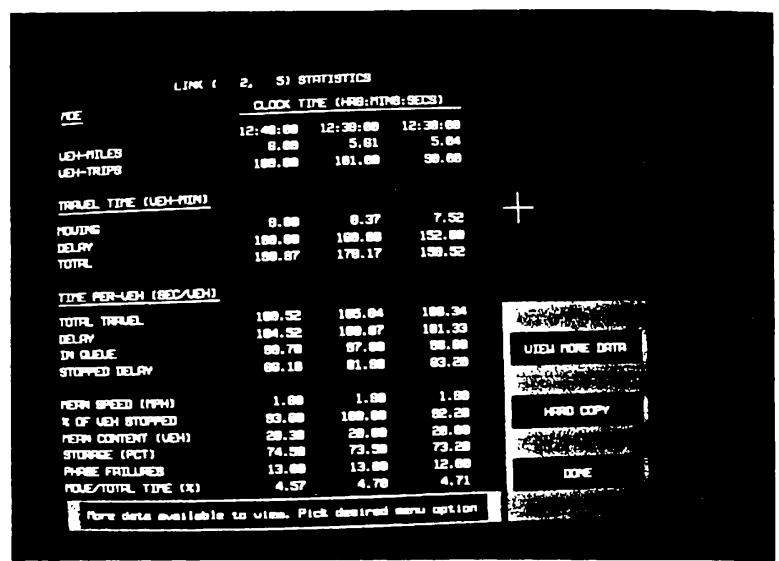

Exhibit 7. Link Statistics (tabular format)

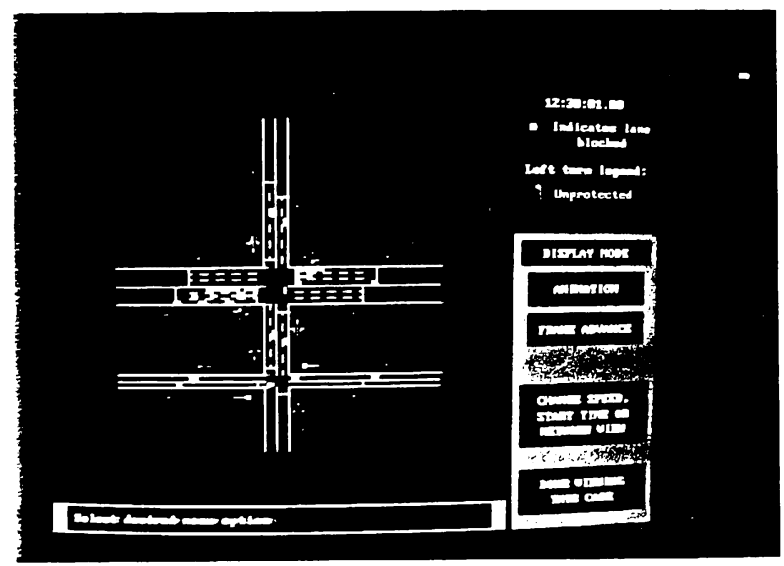

Exhibit 8. Network View with Vehicles

move over time. The signal control is also dynamic, changing from green to yellow to red, over time, and shown by traffic movement.

Generally, a session will begin with the user reviewing the specifications of the traffic environment (i.e., the displays of the NETSIM inputs) to check whether there are any errors. Following a successful review, the user will then focus attention on an examination of the NETSIM outputs. First, the user will probably seek to identify where extremum conditions (i.e., "hotspots") exist and to quantify the extent of any associated problems. It is also likely that the user would seek an overview of the dynamics of traffic flow on the network. While using the "hotspots" feature, the user will probably examine individual links and nodes in detail. There are many other displays, not included herein, which also serve to provide the user with the insights and understanding of traffic operations on the test network. Specifically, the user can view the entire dynamic process of traffic flow using the vehicle animation feature.

At the conclusion of a session, the user may decide to modify the traffic environment in a manner which, in his/her opinion, may improve traffic performance. The user need specify only these changes to the NETSIM input stream by using the ICG input processing software (e.g., TRAF-EDIT--see Figure 1), then repeat the analysis process. In this way, several detailed studies can be undertaken in a day.

\section{CONCLUSIONS}

All transportation planning and traffic engineering tools require significant amounts of information in the form of input data. In addition, as outlined in the Appendix, NETSIM provides the user with an extraordinary amount of invaluable, detailed information which describes traffic operations over space and time. In the past, potential users were discouraged by the labor intensive effort of preparing and processing the input data, and by the effort required to sift through extensive statistical output listings in order to extract the information of interest.

These concerns have now effectively been eliminated by the development of ICG tools on both ends of the information spectrum. Input data may now be entered by technician personnel with no knowledge of computers or of traffic engineering. This rate of data entry is rapid and is subject to the quality assurance features of the software which checks all input data. The NETSIM input data and results are presented in many attractive ICG formats, all easily accessible to users, and requiring no background whatever in computers. The GTRAF design permits the user to immediately identify the problem areas in the traffic environment and to rapidly determine the causal factors contributing to these problems. Thus, the perceived impediments to the use of NETSIM have been removed and the utility of this tool, greatly enhanced.

Based on limited experience with GTRAF to date, it appears to provide users with an efficient -- almost entertaining -- medium for gaining a level of insight into traffic operations and for achieving a level of productivity that is simply without equal. We anticipate that the use of ICG will grow rapidly as an increasing number of agencies become aware of its benefits and as more PC's and workstations with graphics capabilities, are procured.

\section{APPENDIX A. THE NETSIM TRAFFIC SIMULATION MODEL}

The basic approach in the NETSIM model is the application of an interval-scanning, microscopic, stochastic simulation of traffic movements to describe traffic operations. The physical street system is represented as a network comprised of one-way links, with the intersections represented as nodes. The traffic stream is modeled explicitly; each vehicle on the network is treated as an identifiable entity. Furthermore, each vehicle is identified by category (auto, car pool, truck, bus) and by type. Up to 16 different types of vehicles with different operating and performance characteristics may be specified defining the four categories of the vehicle fleet. In addition, a "driver behavior characteristic" (ranging from passive to aggressive) is assigned to each vehicle. A vehicle's kinematic properties (position, speed, acceleration) as well as its status (queued, free-flowing) are determined in each one-second time-step. Turn movements are assigned stochastically, as are free-flow speeds, queue discharge headways and other vehicle-specific behavioral attributes. Consequently, each vehicle's behavior is simulated in a stochastic manner, reflecting real-world processes.

Each time a vehicle is moved by the program logic, its position (both lateral and longitudinal) on a network link is determined, as well as its relationship to other vehicles nearby. This approach allows the program to simulate the detailed, vehicle-specific, traffic processes so that actuated signal controls may be simulated and bus-auto interaction may be explicitly modeled. In general, most conditions experienced on an urban 
traffic environment can be realistically described.

As an interval-scanning simulation model, each vehicle is moved each second according to car-following logic and in response to traffic control devices and other conditions which influence vehicle behavior. The onset of congestion could result in queues extending throughout the length of a link to block the upstream intersection, thus impeding traffic flow there. The effects of such blockage are modeled realistically; vehicles on cross streets "recognize" when they are physically blocked by spillback and cannot move. Pedestrian traffic can delay turning vehicles at intersections.

Many other examples describing the factors which are prevalent in urban environs and which are explicitly treated by NETSIM can be given. Most of these microscopic treatments are transparent to the user, whose prime concern is the "bottom-line" description of traffic operations provided by the model in the form of link-specific, detailed, operational Measures of Effectiveness (MOE).

A wealth of information is provided as output by NETSIM. This list presented below is a sampling of what is available:

- Vehicle-Miles of Travel

- Number of Vehicles Discharged from Link

- Total Vehicle Minutes of Travel Time

- Total Delay Time (computed as the difference between the total travel time and "idealized" travel time for each link based on a specified free speed)

- Ratio of Moving Time to Total Travel Time

- Total Travel Time for Link

- Average Travel Time per Vehicle

- Average Delay Time per Vehicle

- Average Delay Time per Vehicle-Mile

- Percent Stopped Delay

- Average Traffic Speed

- Average Occupancy

- Average Number of Stops per Vehicle (expressed as the percent of vehicles stopping at least once within the link)

- Average Storage Percentage (expressed as a time-average of the length of the link occupied by vehicles divided by its total storage capacity)

- Cycle Failure (defined as the number of times a queue present at the beginning of the green phase fails to clear the stop line by the end of the green)

- History of Queue Lengths, by Lane

- History of Intersection Spillback

The ability to replicate individual vehicle trajectories allows NETSIM to simulate the data accessed by individual passage and presence (long loop) detectors.

\section{REFERENCES}

Andrews, B. and E. Lieberman (1984), "NETSIM Interactive Input - Intraf User's Guide," Final Report under FHWA Contract DTFH61-82-C-00103, KLD Associates, Huntington Station, NY.

Lieberman, E. (1979), "Traffic Simulation: Past, Present and Potential," In Proceedings of the International Symposium on Traffic Control Systems.

Lieberman, E. (1982), Enhanced NETSIM Program, TRB Special Report 194.

Lieberman, E., M. Yedlin, and B. Andrews (1985), "Traf Integrated Simulation Model - Phase II," Final Report, Volumes 1-4, under FHWA Contract DTFH61-85-C-00005, KLD Associates, Huntington Station, NY.
Roberts, G., S. Chin, and L. Till (1985), "Integrated Traffic Data System (ITDS) Programmers Manual," Report prepared under FHWA Contract, Oak Ridge National Laboratory, Oak Ridge, TN.

Rothenberg, M.J. (1985), "Urban Congestion in the United States: What Does the Future Hold?," ITE Journal 55, 7. 